\title{
On the Symmetries and Conservation Laws of the Multidimensional Nonlinear Damped Wave Equations
}

\author{
Usamah S. Al-Ali, ${ }^{1}$ Ashfaque H. Bokhari, ${ }^{1}$ A. H. Kara, ${ }^{2}$ and F. D. Zaman ${ }^{1}$ \\ ${ }^{1}$ Department of Mathematics and Statistics, King Fahd University of Petroleum and Minerals, Dhahran 31261, Saudi Arabia \\ ${ }^{2}$ School of Mathematics, University of the Witwatersrand, Johannesburg, Wits 2050, South Africa \\ Correspondence should be addressed to A. H. Kara; abdul.kara@wits.ac.za
}

Received 8 August 2016; Accepted 1 December 2016; Published 12 January 2017

Academic Editor: Stephen C. Anco

Copyright (C) 2017 Usamah S. Al-Ali et al. This is an open access article distributed under the Creative Commons Attribution License, which permits unrestricted use, distribution, and reproduction in any medium, provided the original work is properly cited.

We carry out a classification of Lie symmetries for the $(2+1)$-dimensional nonlinear damped wave equation $u_{t t}+f(u) u_{t}=$ $\operatorname{div}(g(u) \operatorname{grad} u)$ with variable damping. Similarity reductions of the equation are performed using the admitted Lie symmetries of the equation and some interesting solutions are presented. Employing the multiplier approach, admitted conservation laws of the equation are constructed for some new, interesting cases.

\section{Introduction}

The wave equation is a mathematical model that is used to describe the propagations of waves as they occur in physics. It arises in many fields like acoustics, electromagnetism, fluid mechanics, general relativity, hydrodynamics, and quantum mechanics $[1,2]$. Therefore, investigating the wave equations remains one of the hottest areas of research in applied mathematics.

Historically, the first serious attempt to understand wave behavior dates back to the sixth century BC when Pythagoras studied the properties of sound waves produced by a string in musical instrument [3]. During the scientific revolution, rapid advances in understanding waves were made due to the works of many prominent scientists such as Bynam et al. [4]. Furthermore, a revolutionary change in human perception of wave phenomena took place in the nineteenth century when Maxwell formulated his electromagnetic field theory [5].

In fact, mathematicians focused their attention in investigating the formation and motion of waves. Thus, extensive studies have been conducted to obtain exact solutions to both linear and nonlinear wave equations. In this context, Cajori and Farlow obtained the first exact solution to the linear wave equation $[6,7]$. Further researches have been conducted to investigate the nonlinear wave equations where different approximate and numeric methods have been developed; see, for example, [8-15].

Nevertheless, the classical wave model lacks the ability to predict the behavior of wave phenomena arising in physical systems under certain circumstances. Such situations are frequently met when dealing with waves whose behavior exhibits diffusion phenomena which can only be described using the damped wave equation.

A damped wave in general is a wave whose amplitude of oscillation decreases with time. This damping is expressed mathematically by coupling an extra term to the classical wave equation. The additional term has the effect of controlling the speed of oscillation [16]. Thus, the damped equation model can describe effectively many physical systems involving processes that dissipate the energy stored in the oscillation. In order to understand these physical systems, the damped wave equations need to be investigated.

In fact, many authors have studied different classes of damped wave equations by investigating their solutions or by studying their asymptotic behavior [17-19]. Some other researchers adopted the numerical approach in tackling this type of equations [20]. However, studying damped wave equations in terms of Lie point symmetries admitted by them was not sufficiently addressed in literature. Indeed, attention was focused so far on classifying symmetries of 
classical wave equations. In this context, a lot of work has been published; see, for example, [21-23]. Ibragimov extended this kind of work to the damped wave equations. For example, he studied different forms of unperturbed damped wave equations by classifying their Lie symmetries [23]. Some other researchers implemented the symmetries of certain classes of damped wave equations to obtain analytical solutions [24].

Indeed, one of the main applications of symmetries is the construction of conservation laws for a given system. The link between conservation laws and symmetries was first introduced by Noether in 1918 [25, 26]. Although Noether's theorem provides a very powerful method for obtaining conservation laws, it has a limitation in the sense that it is only applicable for variational PDEs as it requires the existence of a Lagrangian. Therefore, the multiplier approach has been developed for deriving conservation laws for variational and nonvariational PDEs. The procedure of obtaining conservation laws using the multiplier approach is explained in many references; see, for example, [27, 28].

The aim of this paper is to extend the investigation of damped wave equations of Ibragimov [22] by considering the $(2+1)$-dimensional nonlinear damped wave equation given by

$$
u_{t t}+f(u) u_{t}=\operatorname{div}(g(u) \operatorname{grad} u)
$$

where the equation will be classified in terms of Lie point symmetries it admits. These symmetries will be utilized in performing different similarity reductions and, where possible, exact solutions will be obtained. Furthermore, the obtained symmetries will be exploited to construct conservation laws for some cases of interest by applying the multiplier approach.

\section{Symmetry Classification of the Damped Wave Equation}

We will consider (1) which can be equivalently written in the following form:

$$
u_{t t}+f(u) u_{t}=g(u)\left(u_{x x}+u_{y y}\right)+g^{\prime}(u)\left(u_{x}^{2}+u_{y}^{2}\right) .
$$

A symmetry of a differential equation in general is a transformation that keeps its family of solutions invariant. To obtain the symmetry algebra of (2), we take the infinitesimal generator of symmetry algebra of the form

$$
X=\xi \frac{\partial}{\partial x}+\gamma \frac{\partial}{\partial y}+\tau \frac{\partial}{\partial t}+\phi \frac{\partial}{\partial u}
$$

where the coefficients $\xi, \gamma, \tau$, and $\phi$ are functions of $x, y, t$, and $u$. Using the invariance condition, that is, applying the 2nd prolongation $X^{[2]}$ to (2), yields the following system of determining equations:

$$
\begin{aligned}
& \xi_{u}=\gamma_{u}=\tau_{u}=\phi_{u u}=0, \\
& \xi_{t}-g(u) \tau_{x}=0, \\
& \xi_{y}+\gamma_{x}=0, \\
& \gamma_{t}-g(u) \tau_{y}=0, \\
& f_{u} \phi+f(u) \tau_{t}-\tau_{t t}+g(u) \tau_{x x}+g(u) \tau_{y y}+2 \phi_{t u}=0, \\
& -f(u) \gamma_{t}-\gamma_{t t}+g(u) \gamma_{x x}+g(u) \gamma_{y y}-2 g(u) \phi_{y u} \\
& \quad=0, \\
& -f(u) \xi_{t}-\xi_{t t}+g(u) \xi_{x x}+g(u) \xi_{y y}-2 g(u) \phi_{x u} \\
& \quad=0, \\
& f(u) \phi_{t}+\phi_{t t}-g(u) \phi_{x x}-g(u) \phi_{y y}=0, \\
& -g_{u} \phi+2 g(u) \xi_{x}-2 g(u) \tau_{t}=0, \\
& -g_{u} \phi+2 g(u) \gamma_{y}-2 g(u) \tau_{t}=0 .
\end{aligned}
$$

Since we are interested in classifying Lie symmetries and corresponding solutions of (2), we start by writing (13) in the form

$$
\frac{g_{u}}{g} \phi=2 \gamma_{y}-2 \tau_{t}
$$

From the above equation, two cases are considered; namely, $\phi=0$ and $\phi \neq 0$. We discuss each case separately as follows.

Case $1(\phi=0)$. Substituting $\phi=0$ in the overdetermined system, (4)-(13), leads to the following solution:

$$
\begin{aligned}
& \tau=c_{1}, \\
& \xi=c_{2}-c_{4} y, \\
& \gamma=c_{3}+c_{4} x .
\end{aligned}
$$

The above solution is valid for arbitrary $f$ and arbitrary $g$ and consequently leads to the minimal subalgebra group $\langle G 4\rangle$ of vector fields $\langle\partial / \partial t, \partial / \partial x, \partial / \partial y, x(\partial / \partial y)-y(\partial / \partial x)\rangle$.

Case $2(\phi \neq 0)$. In this case we can reconsider (14) by writing in the form

$$
\frac{g_{u}}{g}=\frac{2 \gamma_{y}-2 \tau_{t}}{\phi}
$$

Since the left hand side of (16) depends only on $u$, it can be put in the form

$$
\frac{g_{u}}{g}=\frac{2 \gamma_{y}-2 \tau_{t}}{\phi}=h(u) .
$$


At this stage, we consider three possibilities; namely, $h(u)=0$, $h(u)=$ constant $\neq 0$, and $h(u)$ functionally depending on $u$.

Case $2.1(h(u)=0)$. In this case, (17) can be put in the form

$$
\frac{g_{u}}{g}=\frac{2 \gamma_{y}-2 \tau_{t}}{\phi}=0 .
$$

From the above equation it is instantly found that $g(u)=\alpha$ while $\gamma_{y}=\tau_{t}$. Substituting for $g$ in (10) and differentiating the resulting equation with respect to $u$ give

$$
f_{u} \xi_{t}=0
$$

Thus, three cases arise from (19); namely, (1) $f_{u} \neq 0, \xi_{t}=0$, (2) $f_{u}=0, \xi_{t} \neq 0$, and (3) $f_{u}=\xi_{t}=0$, which we consider one by one.

Case 2.1.1 $\left(f_{u} \neq 0, \xi_{t}=0\right)$. Differentiating (8) with respect to $u$ gives

$$
f_{u u} \phi+f_{u} \phi_{u}+f_{u} \tau_{t}=0
$$

Since $f_{u} \neq 0$, the above equation can be written as

$$
\frac{f_{u u}}{f_{u}}=-\frac{\phi_{u}+\tau_{t}}{\phi}=G(u) .
$$

Notice that (21) generates three possibilities; $G(u)=0, G(u)=$ constant $\neq 0$, and $G^{\prime}(u) \neq 0$.

Case 2.1.1a $(G(u)=0)$. Hence, $(21)$ becomes

$$
\frac{f_{u u}}{f_{u}}=-\frac{\phi_{u}+\tau_{t}}{\phi}=0 .
$$

Solving (22) and substituting the solution in the overdetermined system (see (4)-(13)) produce the following solution:

$$
\begin{aligned}
& \tau=c_{1}+c_{5} t, \\
& \xi=c_{2}+c_{5} x-c_{4} y, \\
& \gamma=c_{3}+c_{4} x+c_{5} y, \\
& \phi=-c_{5}\left(\frac{\sigma}{\beta}+u\right),
\end{aligned}
$$

with $f(u)=\beta u+\sigma$ such that $\beta \neq 0$. The generators of the five-dimensional symmetry group are generated by vector fields given by $\langle\partial / \partial t, \partial / \partial x, \partial / \partial y, x(\partial / \partial y)-y(\partial / \partial x), t(\partial / \partial t)+$ $x(\partial / \partial x)+y(\partial / \partial y)-(\sigma / \beta+u)(\partial / \partial u)\rangle$.

Case 2.1.1b $(G(u)=\beta \neq 0)$. In this case (21) takes the form

$$
\frac{f_{u u}}{f_{u}}=-\frac{\phi_{u}+\tau_{t}}{\phi}=\beta .
$$

Following the method adopted in Case 2.1.1a leads to the following solution of the overdetermined system equations (4)-(13):

$$
\begin{aligned}
& \tau=c_{1}-c_{5} t, \\
& \xi=c_{2}-c_{5} x-c_{4} y, \\
& \gamma=c_{3}+c_{4} x-c_{5} y, \\
& \phi=\frac{c_{5}}{\beta},
\end{aligned}
$$

with $f(u)=(\sigma / \beta) e^{\beta u}$ and $\sigma \neq 0$ and $\beta \neq 0$. The corresponding algebra of the 5 -symmetry group is spanned by $\langle\partial / \partial t, \partial / \partial x, \partial / \partial y, x(\partial / \partial y)-y(\partial / \partial x),-t(\partial / \partial t)-x(\partial / \partial x)-$ $y(\partial / \partial y)+(1 / \beta)(\partial / \partial u)\rangle$

Case 2.1.1c $\left(G^{\prime}(u) \neq 0\right)$. To deal with this case, we first differentiate (21) with respect to $u$ to obtain

$$
G(u) \phi_{u}+G^{\prime}(u) \phi=0 .
$$

It is straightforward to solve the above equation to get

$$
\phi=\frac{A(t, x, y)}{G(u)} .
$$

Substituting (27) in (4) yields

$$
G(u)=\frac{1}{\beta u+\rho} .
$$

Hence, (27) becomes

$$
\phi=A(t, x, y)(\beta u+\rho) .
$$

Also, (21) can be recast as

$$
\frac{f_{u u}}{f_{u}}=\frac{1}{\beta u+\rho} .
$$

Solving the above equation immediately yields

$$
f(u)=\frac{\sigma}{\beta+1}(\beta u+\rho)^{1+1 / \beta},
$$

where $\beta \notin\{0,-1\}$. After some more manipulation, the following solution of the overdetermined system equations (4)-(13) is obtained:

$$
\begin{aligned}
& \tau=c_{1}+c_{5} t, \\
& \xi=c_{2}+c_{5} x-c_{4} y, \\
& \gamma=c_{3}+c_{4} x+c_{5} y, \\
& \phi=-\frac{c_{5}}{\beta+1}(\beta u+\rho),
\end{aligned}
$$

with $g(u)=\alpha$. Accordingly, the vector fields generating a fiveparameter Lie group are given by $\langle\partial / \partial t, \partial / \partial x, \partial / \partial y, x(\partial / \partial y)-$ 
$y(\partial / \partial x), t(\partial / \partial t)+x(\partial / \partial x)+y(\partial / \partial y)-(1 /(\beta+1))(\beta u+$ $\rho)(\partial / \partial u)\rangle$.

Case 2.1.2 $\left(f_{u}=0, \xi_{t} \neq 0\right)$. In this case, $f(u)=\beta$. Hence, since both functions $f$ and $g$ are constant; then we expect this case to give the maximal set of Lie symmetries. After carrying out some calculations it turns out that the obtained solution of the determining system is

$$
\begin{aligned}
\tau & =c_{1}+2 c_{5} x+2 c_{6} y, \\
\xi & =c_{2}+2 c_{5} \alpha t-c_{4} y, \\
\gamma & =c_{3}+2 c_{6} \alpha t+c_{4} x, \\
\phi & =\left(c_{7}-c_{5} \beta x-c_{6} \beta y\right) u+A(t, x, y),
\end{aligned}
$$

where $A(t, x, y)$ satisfies the differential constraint given by

$$
\beta A_{t}+A_{t t}-\alpha A_{x x}-\alpha A_{y y}=0 .
$$

As a result, this case admits the 7-group Lie symmetries given by $\langle\partial / \partial t, \partial / \partial x, \partial / \partial y, x(\partial / \partial y)-y(\partial / \partial x), 2 x(\partial / \partial t)+$ $2 \alpha t(\partial / \partial x)-\beta x u(\partial / \partial u), 2 y(\partial / \partial t)+2 \alpha t(\partial / \partial y)-\beta y u(\partial / \partial u)$, $u(\partial / \partial u)\rangle$ along with an arbitrary symmetry $A(t, x, y)(\partial / \partial u)$.

Case 2.1.3 $\left(f_{u}=\xi_{t}=0\right)$. It turns out that this case leads to finite six-dimensional symmetry algebra which is a subset of the algebra obtained in Case 2.1.2.

Case $2.2(h(u)=$ constant $\neq 0)$. In this case (17) takes the form

$$
\frac{g_{u}}{g}=\frac{2 \gamma_{y}-2 \tau_{t}}{\phi}=\alpha,
$$

which can be easily integrated over $u$ to give

$$
g(u)=\beta e^{\alpha u} .
$$

Also, solving (35) for $\phi$ yields

$$
\phi=A(t, x, y) .
$$

Using (37) in the overdetermined system, (4)-(13), and after manipulating further leads to the following solution:

$$
\begin{aligned}
& \tau=c_{1}+c_{5} t, \\
& \xi=c_{2}-c_{4} y+c_{5}\left(1-\frac{\alpha}{2 \sigma}\right) x, \\
& \gamma=c_{3}+c_{4} x+c_{5}\left(1-\frac{\alpha}{2 \sigma}\right) y, \\
& \phi=-\frac{c_{5}}{\sigma},
\end{aligned}
$$

subject to $f(u)=(\rho / \sigma) e^{\sigma u}$, where $\sigma \notin\{0, \alpha / 2\}$. The symmetry algebra for this case is generated by the following five vector fields $\langle\partial / \partial t, \partial / \partial x, \partial / \partial y, x(\partial / \partial y)-y(\partial / \partial x), t(\partial / \partial t)+$ $(1-\alpha / 2 \sigma) x(\partial / \partial x)+(1-\alpha / 2 \sigma) y(\partial / \partial y)-(1 / \sigma)(\partial / \partial u)\rangle$.

Case $2.3\left(h^{\prime}(u) \neq 0\right)$. In this case, we write (17) as

$$
\phi h(u)=2 \gamma_{y}-2 \tau_{t} .
$$

Differentiating the above equation with respect to $u$ and solving immediately give

$$
\phi=\frac{A(t, x, y)}{h(u)},
$$

where $A(t, x, y)$ is an integration function. Substituting (40) in (4) gives

$$
h(u)=\frac{1}{\alpha u+\beta} .
$$

Consequently, (40) becomes

$$
\phi=A(t, x, y)(\alpha u+\beta) .
$$

After some more manipulations, (42) reduces to

$$
\phi=A(t)(\alpha u+\beta) .
$$

Using (41) in (17) gives

$$
\frac{g_{u}}{g}=\frac{1}{\alpha u+\beta},
$$

which on integration over $u$ becomes

$$
g(u)=\lambda(\alpha u+\beta)^{1 / \alpha} .
$$

Furthermore, simple calculations show that $\tau$ is a function of $t$ only. At this point, substituting (43) in (8) and differentiating the resulting equation with respect to $u$ we obtain

$$
f_{u u}(\alpha u+\beta) A(t)=\left(-\alpha A(t)-\tau_{t}\right) f_{u} .
$$

At this stage we consider two possibilities; namely, $f_{u} \neq 0$ and $f_{u}=0$.

Case 2.3.1 $\left(f_{u} \neq 0\right)$. After some manipulations the following solution is obtained:

$$
\begin{aligned}
\tau & =c_{1}-\frac{2 c_{5}(\alpha+\sigma)}{1-2(\alpha+\sigma)} t, \\
\xi & =c_{2}-c_{4} y+c_{5} x, \\
\gamma & =c_{3}+c_{4} x+c_{5} y, \\
\phi & =\frac{2 c_{5}}{1-2(\alpha+\sigma)}(\alpha u+\beta),
\end{aligned}
$$

where $f(u)=(\rho /(\sigma+\alpha))(\alpha u+\beta)^{\sigma / \alpha+1}$, provided that $\sigma+$ $\alpha \neq 0$ and $\alpha \neq 0$. As a result, the symmetry algebra for this case is constructed, generating a five-parameter group $\langle\partial / \partial t, \partial / \partial x, \partial / \partial y, x(\partial / \partial y)-y(\partial / \partial x),-(2(\alpha+\sigma) /(1-2(\alpha+$ 
$\sigma)) t(\partial / \partial t)+x(\partial / \partial x)+y(\partial / \partial y)+(2(\alpha u+\beta) /(1-2(\alpha+$ $\sigma))(\partial / \partial u)\rangle$.

Case 2.3.2 $\left(f^{\prime}(u)=0\right)$. This case suggests that $f(u)=\sigma$. Then, carrying out further calculations, one arrives at the following results:

$$
\begin{aligned}
\tau & =\left(c_{5}-\frac{c_{7}}{2}\right) t-\frac{c_{6}}{2 \sigma^{2}} e^{-\sigma t}+c_{1}, \\
\xi & =c_{5} x-c_{4} y+c_{2}, \\
\gamma & =c_{4} x+c_{5} y+c_{3}, \\
\phi & =\left(c_{7}-\frac{c_{6}}{\sigma} e^{-\sigma t}\right)(\alpha u+\beta) .
\end{aligned}
$$

Substituting (48) in (8) yields

$$
\sigma\left(c_{5}-\frac{c_{7}}{2}\right)+c_{6} e^{-\sigma t}(1+2 \alpha)=0 .
$$

It is obvious that (49) leads to the following two equations:

$$
\begin{aligned}
c_{7} & =2 c_{5}, \\
c_{6}(1+2 \alpha) & =0 .
\end{aligned}
$$

As a result, (51) gives rise to two possibilities. Namely, $\alpha=$ $-1 / 2$ and $c_{6}=0$.

Case 2.3.2a $\left(\alpha=-1 / 2, c_{6} \neq 0\right)$. Substituting (50) and the value of $\alpha$ in (48) gives

$$
\begin{aligned}
& \tau=c_{1}-\frac{c_{6}}{2 \sigma^{2}} e^{-\sigma t}, \\
& \xi=c_{2}-c_{4} y+c_{5} x, \\
& \gamma=c_{3}+c_{4} x+c_{5} y, \\
& \phi=\left(2 c_{5}-\frac{c_{6}}{\sigma} e^{-\sigma t}\right)\left(\beta-\frac{1}{2} u\right) .
\end{aligned}
$$

Consequently, the symmetry algebra is extended by two extra symmetries: $\langle\partial / \partial t, \partial / \partial x, \partial / \partial y, x(\partial / \partial y)-y(\partial / \partial x), x(\partial / \partial x)+$ $y(\partial / \partial y)+2(\beta-(1 / 2) u)(\partial / \partial u),-\left(e^{-\sigma t} / 2 \sigma^{2}\right)(\partial / \partial t)-\left(e^{-\sigma t} /\right.$ $\sigma)(\beta-(1 / 2) u)(\partial / \partial u)\rangle$, where $\sigma \neq 0$. Notice also that (45) in this case takes the form $g(u)=\lambda(\beta-(1 / 2) u)^{-2}$.

Case 2.3.2b $\left(\alpha \neq-1 / 2, c_{6}=0\right)$. The solution of the overdetermined system for this case takes the form

$$
\begin{aligned}
& \tau=c_{1}, \\
& \xi=c_{2}-c_{4} y+c_{5} x, \\
& \gamma=c_{3}+c_{4} x+c_{5} y, \\
& \phi=2 c_{5}(\alpha u+\beta),
\end{aligned}
$$

which generates a five-dimensional symmetry group $\langle\partial / \partial t$, $\partial / \partial x, \partial / \partial y, x(\partial / \partial y)-y(\partial / \partial x), x(\partial / \partial x)+y(\partial / \partial y)+2(\alpha u+$ $\beta)(\partial / \partial u)\rangle$. Finally, our calculations have shown that the case $\alpha=-1 / 2$ with $c_{6}=0$ is not interesting as it does not generate extra symmetries.

At this stage, we conclude Section 2 where it is shown that (2) has at least four-dimensional symmetry algebra which is given by the group $\langle G 4\rangle$. In this section we have presented the particular forms of $f$ and $g$ that admit extra symmetries. Table 1 shows the extra symmetries obtained for each case arising in the classification.

\section{Reduction of the Damped Wave Equation}

In this section we briefly discuss solutions of considered damped wave equation (2) by reduction via symmetry algebra. In particular, we will use two-dimensional subalgebras to reduce the considered equation to an ODE. The procedure of reducing a PDE using its symmetries is standard and it is explained in detail in many references; see, for example, [29]. In the context of this paper, the procedure of performing the reduction will be shown in detail for the general form of (2) where the functions $f(u)$ and $g(u)$ are arbitrary. For particular forms of $f(u)$ and $g(u)$, the reduced equations and the corresponding similarity variables are given in Table 2.

Notice that the commutation relations between elements of $\langle G 4\rangle$ are $\left[X_{1}, X_{2}\right]=\left[X_{1}, X_{3}\right]=\left[X_{1}, X_{4}\right]=\left[X_{2}, X_{3}\right]=0$. Thus, we have four subalgebras of two dimensions. We will perform reduction for each subalgebra as follows.

(a) Reduction under the Subalgebra $\left\langle X_{1}, X_{2}\right\rangle$. Solving the characteristic system, it is straightforward to find the following similarity variables:

$$
\begin{gathered}
\xi_{1}(t, x, y)=x, \\
\xi_{2}(t, x, y)=y, \\
V\left(\xi_{1}, \xi_{2}\right)=u .
\end{gathered}
$$

In the light of these similarity transformations, (2) is easily reduced to the form

$$
g(V)\left(V_{\xi_{1} \xi_{1}}+V_{\xi_{2} \xi_{2}}\right)+g^{\prime}(V)\left(V_{\xi_{1}}^{2}+V_{\xi_{2}}^{2}\right)=0
$$

The second level of reduction is performed using the symme$\operatorname{try} X_{2}=\partial / \partial x$. Hence, the symmetry

$$
X=X_{2}\left(\xi_{1}\right) \frac{\partial}{\partial \xi_{1}}+X_{2}\left(\xi_{2}\right) \frac{\partial}{\partial \xi_{2}}+X_{2}(V) \frac{\partial}{\partial V}
$$

is inherited by (55). This inherited symmetry is used to obtain the new similarity variables. Thus, again, we solve the characteristic system to obtain the following similarity variables:

$$
\begin{gathered}
s\left(\xi_{1}, \xi_{2}\right)=\xi_{2}, \\
W(s)=V .
\end{gathered}
$$

Using the new similarity variables, (55) is reduced to

$$
g(W) W_{s s}+g^{\prime}(W) W_{s}^{2}=0
$$


TABLE 1: Symmetries other than minimal of (2) for particular forms of $f$ and $g$.

\begin{tabular}{|c|c|c|c|}
\hline Case number & $f(u)$ & $g(u)$ & Symmetries other than minimal \\
\hline 1 & Arbitrary & Arbitrary & No extra symmetries \\
\hline 2 & $\beta u+\sigma$ & $\alpha$ & $X_{5}=t \frac{\partial}{\partial t}+x \frac{\partial}{\partial x}+y \frac{\partial}{\partial y}-\left(\frac{\sigma}{\beta}+u\right) \frac{\partial}{\partial u}$ \\
\hline 3 & $\frac{\sigma}{\beta} e^{\beta u}$ & $\alpha$ & $X_{5}=-t \frac{\partial}{\partial t}-x \frac{\partial}{\partial x}-y \frac{\partial}{\partial y}+\frac{1}{\beta} \frac{\partial}{\partial u}$ \\
\hline 4 & $\frac{\sigma}{\beta+1}(\beta u+\rho)^{1+1 / \beta}$ & $\alpha$ & $X_{5}=t \frac{\partial}{\partial t}+x \frac{\partial}{\partial x}+y \frac{\partial}{\partial y}-\frac{1}{\beta+1}(\beta u+\rho) \frac{\partial}{\partial u}$ \\
\hline 5 & 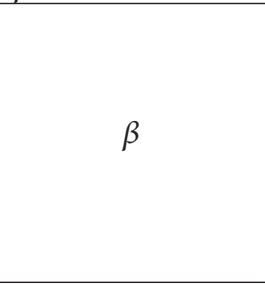 & $\alpha$ & $\begin{array}{c}X_{5}=2 x \frac{\partial}{\partial t}+2 \alpha t \frac{\partial}{\partial x}-\beta x u \frac{\partial}{\partial u} \\
X_{6}=2 y \frac{\partial}{\partial t}+2 \alpha t \frac{\partial}{\partial x}-\beta y u \frac{\partial}{\partial u} \\
X_{7}=u \frac{\partial}{\partial u} \\
X_{\infty}=A(t, x, y) \frac{\partial}{\partial u}, \quad \text { where } A(t, x, y) \text { satisfies (2) }\end{array}$ \\
\hline 6 & $\frac{\rho}{\sigma} e^{\sigma u}$ & $\beta e^{\alpha u}$ & $X_{5}=t \frac{\partial}{\partial t}+\left(1-\frac{\alpha}{2 \sigma}\right) x \frac{\partial}{\partial x}+\left(1-\frac{\alpha}{2 \sigma}\right) y \frac{\partial}{\partial y}-\frac{1}{\sigma} \frac{\partial}{\partial u}$ \\
\hline 7 & $\frac{\rho}{\alpha+\sigma}(\alpha u+\beta)^{\sigma / \alpha+1}$ & $\lambda(\alpha u+\beta)^{1 / \beta}$ & $X_{5}=\frac{-2(\alpha+\sigma)}{1-2(\alpha+\sigma)} t \frac{\partial}{\partial t}+x \frac{\partial}{\partial x}+y \frac{\partial}{\partial y}+\frac{2(\alpha u+\beta)}{1-2(\alpha+\sigma)} \frac{\partial}{\partial u}$ \\
\hline 8 & 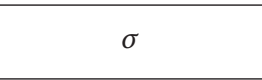 & $\lambda(\alpha u+\beta)^{1 / \alpha}$ & $X_{5}=x \frac{\partial}{\partial x}+y \frac{\partial}{\partial y}+2(\alpha u+\beta) \frac{\partial}{\partial u}$ \\
\hline 9 & $\sigma$ & $\lambda(\beta-0.5 u)^{-2}$ & $\begin{array}{l}X_{5}=x \frac{\partial}{\partial x}+y \frac{\partial}{\partial y}+2(\beta-0.5 u) \frac{\partial}{\partial u} \\
X_{6}=\frac{-e^{-\sigma t}}{2 \sigma^{2}} \frac{\partial}{\partial t}-\frac{e^{-\sigma t}}{\sigma}(\beta-0.5 u) \frac{\partial}{\partial u}\end{array}$ \\
\hline
\end{tabular}

(b) Reduction under the Subalgebra $\left\langle X_{1}, X_{3}\right\rangle$. Following the same procedure as in case (a) we reduce (2) again into (58) but with the following similarity variables: $s=x$ and $W=u$.

(c) Reduction under the Subalgebra $\left\langle X_{1}, X_{4}\right\rangle$. The damped wave equation, that is (2), in this case is reduced to

$$
g(W)\left(W_{s}+s W_{s s}\right)+s^{2} g^{\prime}(W) W_{s}^{2}=0
$$

The corresponding similarity variables are $s=x^{2}+y^{2}$ and $W=u$.

(d) Reduction under the Subalgebra $\left\langle X_{2}, X_{3}\right\rangle$. In this case (2) is reduced to

$$
W_{s s}+f(W) W_{s}=0,
$$

where $s=t$ and $W=u$.

Since reductions performed are valid for arbitrary functions $f(u)$ and $g(u)$, we can use them to obtain exact solutions for all particular forms of $f$ and $g$ arising from the classification. For example, if we consider the case where $f(u)=(\rho / \sigma) e^{\sigma u}$ and $g(u)=\beta e^{\alpha u}$, then we can obtain exact solution for damped wave equation (2) by first substituting for $g$ in (59) to obtain

$$
\beta e^{\alpha W}\left(W_{s}+s W_{s s}\right)+\alpha \beta e^{\alpha W} s^{2} W_{s}^{2}=0 .
$$

Then, solving (61) we get

$$
W=c_{1} \log |s|-c_{1} \log \left|a s+\frac{1}{c_{1}}\right|+c_{2} .
$$

Finally, in order to express the solution in terms of the original variables of (2), we transform each similarity variable in (62) to its corresponding variable given in case (c) above; that is, we substitute for $s=x^{2}+y^{2}$ and $W=u$ in solution (62). This substitution gives rise to a static exact solution of (2), given by

$$
\begin{aligned}
u(t, x, y)= & c_{1} \log \left(x^{2}+y^{2}\right)-c_{1} \log \left|a x^{2}+a y^{2}+\frac{1}{c_{1}}\right| \\
& +c_{2} .
\end{aligned}
$$

Furthermore, we can perform more reductions by considering combination of symmetries. For instance, if we use the subalgebra $\langle\partial / \partial t+2(\partial / \partial x)+\partial / \partial y, \partial / \partial t+\partial / \partial x+2(\partial / \partial y)\rangle$, then (2) is reduced into

$$
9 W_{s s}-3 f(W) W_{s}=2 g(W) W_{s s}+2 g^{\prime}(W) W_{s}^{2},
$$

where $s=x+y-3 t$ and $W=u$. In particular, if we choose $f(u)=e^{u}$ and $g(u)=\sigma$ then (64) becomes

$$
(9-2 \sigma) W_{s s}-3 e^{W} W_{s}=0 .
$$

The solution of $(65)$ is given by $W=\log \left|(3-(2 / 3) \sigma) /\left(c_{1}-s\right)\right|$. This solution is equivalent to the following solution of (2):

$$
u(t, x, y)=\log \left|\frac{3-(2 / 3) \sigma}{c_{1}-x-y+3 t}\right| .
$$

Furthermore, if we choose $f(u)=u$ and $g(u)=\sigma$ then (64) becomes

$$
(9-2 \sigma) W_{s s}-3 W W_{s}=0 .
$$




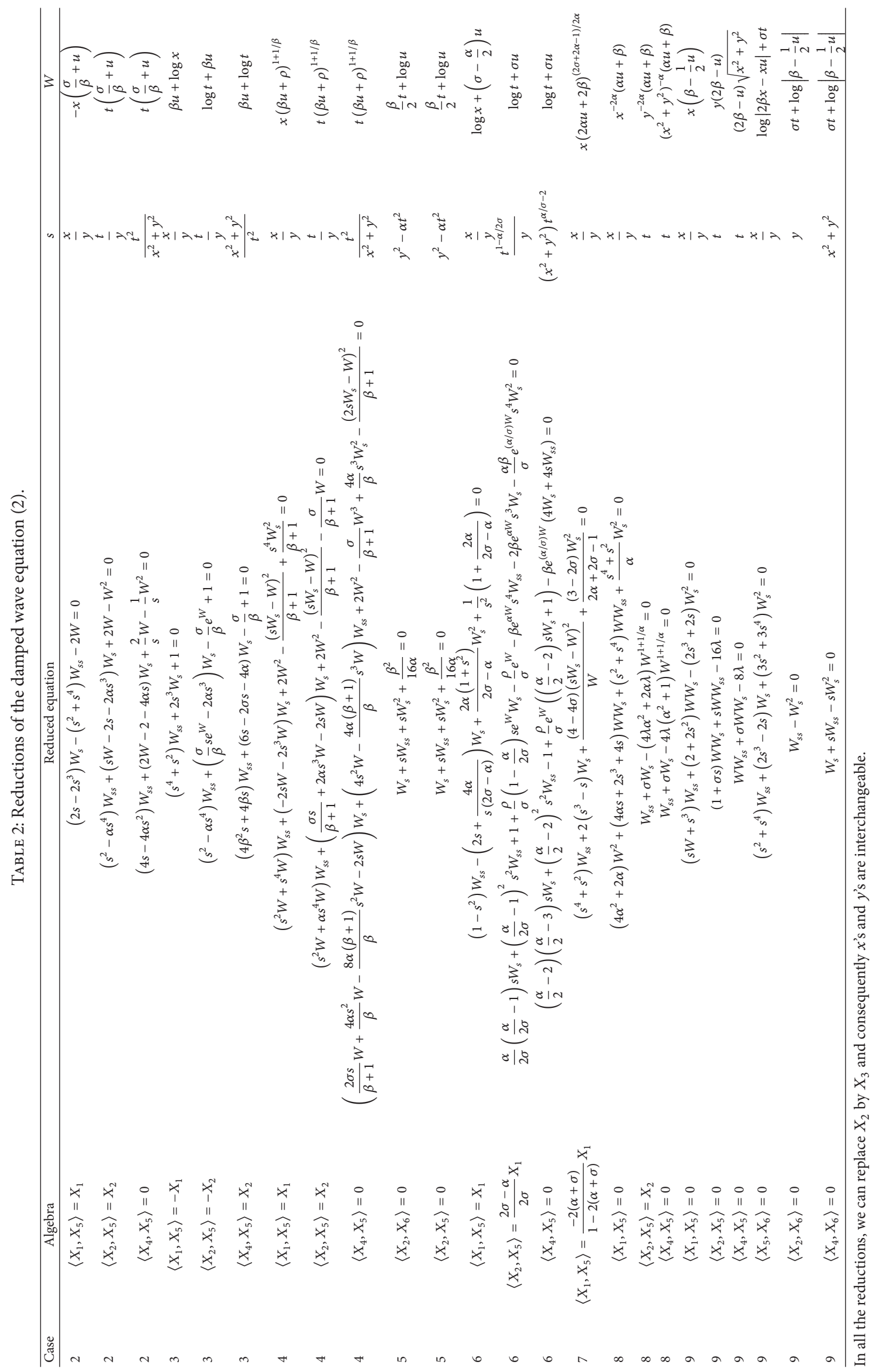


Solving (67) we obtain $W=\sqrt{2 c_{1} / 3} \tan \left[\left(\sqrt{6 c_{1}} / 2(9-2 \sigma)\right) s+\right.$ $C_{2}$ ], which gives rise to the following exact solution of (2):

$$
\begin{aligned}
& u(t, x, y) \\
& \quad=\sqrt{\frac{2 c_{1}}{3}} \tan \left[\frac{\sqrt{6 c_{1}}}{2(9-2 \sigma)}(x+y-3 t)+C_{2}\right] .
\end{aligned}
$$

Exact solutions for different forms of the studied equation are provided in Table 3.

\section{Conservation Laws}

A conservation law of a system of PDEs in general is a divergence expression which vanishes on solutions of the system. Keeping in mind that (2) does not possess a Lagrangian, we implement the alternate multiplier approach to construct conservation laws for some interesting cases that have been obtained in Section 2. This powerful approach can be summarized in two steps as follows.

(i) Determine a set of conservation laws multipliers so that a linear combination of the PDEs with the multipliers yields a divergence expression.

(ii) Find the corresponding flux/densities to construct the conservation laws.

A conserved form of a partial differential equation

$$
E\left(x, y, t, u, u_{x}, u_{y}, u_{t}, u_{x x}, \ldots\right)=0
$$

is represented by a two-form differential operator $\omega=$ $T^{t} D x D y+T^{x} D y D t+T^{y} D t D x$ for which $D \omega$ is a three-form operator which vanishes on the solutions of the PDE ( $D$ is the total exterior derivative). The multiplier method to determine these is detailed in [28], inter alia.

In the remaining part of this paper we will briefly give the multipliers and the corresponding conservation laws resulting from the multiplier approach. The cases will be presented in the same order followed in Section 2 where for each of the cases $T^{x}$ and $T^{y}$ denotes the conserved fluxes in $x$ and $y$ directions, respectively, while $T^{t}$ denotes the conserved densities.

Case 1. This general case admits nine distinct multipliers. In particular, the multipliers $Q_{1}=1$ and $Q_{2}=f_{1}(y-i x)+$ $f_{2}(y+i x)$ are admitted. That is, in terms of real valued functions, we get $Q_{A, B}^{x}=e^{ \pm x}(A \cos y+B \sin y)$ and $Q_{A, B}^{y}=$ $e^{ \pm y}(A \cos x+B \sin x)$. Notice that we have taken into account that all $x$ 's and $y$ 's are interchangeable for this general case.
For the remaining cases we will show the extra multipliers and their corresponding conserved vectors.

Case 2. This case admits the multiplier $Q_{1}=e^{-x}(a \sin y+$ $b \cos y)$. The corresponding conserved flow is given by

$$
\begin{aligned}
T^{x} & =\alpha\left(-e^{-x}\right)(a \sin y+b \cos y)\left(u+u_{x}\right), \\
T^{y} & =\alpha e^{-x}\left(a u \cos y-b u \sin y-a u_{y} \sin y\right. \\
& \left.-b u_{y} \cos y\right), \\
T^{t} & =\frac{1}{2} e^{-x}(a \sin y+b \cos y)\left(\beta u^{2}+2 \sigma u+2 u_{t}\right) .
\end{aligned}
$$

Furthermore, the multiplier $Q_{2}=e^{x}(a \sin y+b \cos y)$ is also admitted. It gives rise to the following conserved current:

$$
\begin{aligned}
& T^{x}=\alpha e^{x}(a \sin y+b \cos y)\left(u-u_{x}\right), \\
& T^{y} \\
& =\alpha e^{x}\left(a u \cos y-b u \sin y-a u_{y} \sin y-b u_{y} \cos y\right), \\
& T^{t}=\frac{1}{2} e^{x}(a \sin y+b \cos y)\left(\beta u^{2}+2 \sigma u+2 u_{t}\right) .
\end{aligned}
$$

Case 3. We have two multipliers for this case. The first one is $Q_{1}=e^{-x}(a \sin y+b \cos y)$. The corresponding conserved vector is given by

$$
\begin{aligned}
T^{x} & =-\alpha e^{-x}(a \sin y+b \cos y)\left(u+u_{x}\right), \\
T^{y} & =\alpha e^{-x}\left(a u \cos y-b u \sin y-a u_{y} \sin y\right. \\
& \left.-b u_{y} \cos y\right),
\end{aligned}
$$

$$
T^{t}=\frac{1}{\beta}\left(e^{-x}(a \sin y+b \cos y)\left(\sigma e^{u}-\sigma+\beta u_{t}\right)\right) .
$$

The second multiplier is $Q_{2}=e^{x}(a \sin y+b \cos y)$ with corresponding conserved vector

$$
\begin{aligned}
& T^{x}=\alpha e^{x}(a \sin y+b \cos y)\left(u-u_{x}\right), \\
& T^{y} \\
& =\alpha e^{x}\left(a u \cos y-b u \sin y-a u_{y} \sin y-b u_{y} \cos y\right), \\
& T^{t}=\frac{1}{\beta}\left(e^{x}(a \sin y+b \cos y)\left(\sigma e^{u}-\sigma+\beta u_{t}\right)\right) .
\end{aligned}
$$

Case 4. This case admits two multipliers. The first one is $Q_{1}=$ $e^{-x}(a \sin y+b \cos y)$. It leads to the following conserved flow:

$$
\begin{aligned}
T^{x} & =-\alpha \beta e^{-x}(a \sin y+b \cos y)\left(u_{x} e^{u}+e^{u}-1\right), \\
T^{y} & =-\alpha \beta e^{-x}\left(u_{y} e^{u}(a \sin y+b \cos y)\right. \\
& \left.-\left(e^{u}-1\right)(a \cos y-b \sin y)\right), \\
T^{t} & =\frac{1}{\sigma^{2}}\left(a \sin y e^{-x}+b \cos y e^{-x}\right)\left(\rho e^{\sigma u}-\rho+\sigma^{2} u_{t}\right) .
\end{aligned}
$$


TABLE 3: Some exact solutions of wave equation (2) with particular forms of $f$ and $g$.

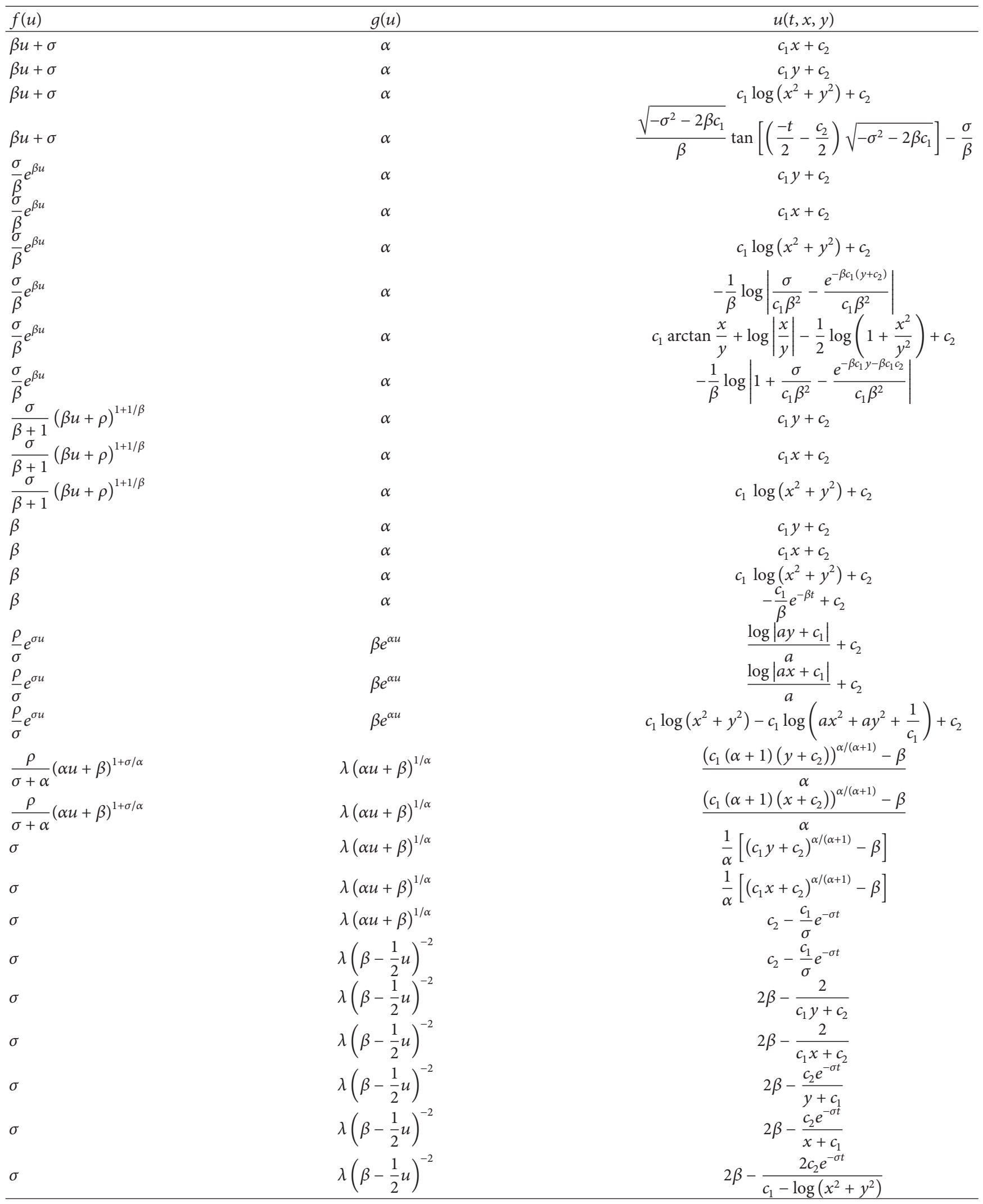


The second multiplier is $Q_{2}=e^{x}(a \sin y+b \cos y)$. It gives rise to the following conserved flow:

$$
\begin{aligned}
T^{x} & =-\alpha \beta(a \sin y+b \cos y)\left(\left(u_{x}-1\right) e^{u+x}+e^{x}\right), \\
T^{y} & =-\alpha \beta e^{x}\left[e ^ { u } \left(u_{y}(a \sin y+b \cos y)-a \cos y\right.\right. \\
& +b \sin y)+a \cos y-b \sin y], \\
T^{t} & =\frac{1}{\sigma^{2}}\left(a e^{x} \sin y+b e^{x} \cos y\right)\left(\rho e^{\sigma u}-\rho+\sigma^{2} u_{t}\right) .
\end{aligned}
$$

Case 5. This case admits two multipliers given by

$$
\begin{aligned}
& Q_{1}=e^{-x}(a \sin y+b \cos y), \\
& Q_{2}=e^{x}(a \sin y+b \cos y),
\end{aligned}
$$

from which the conservation law is too tedious to construct.

Case 6. We have four multipliers given by

$$
\begin{aligned}
& Q_{1}=e^{-x}(a \sin y+b \cos y), \\
& Q_{2}=e^{x}(a \sin y+b \cos y), \\
& Q_{3}=e^{\sigma t+x}(a \sin y+b \cos y), \\
& Q_{4}=e^{\sigma t-x}(a \sin y+b \cos y) .
\end{aligned}
$$

In particular, the fourth multiplier $Q_{4}$ gives rise to the following conserved flow:

$$
\begin{aligned}
T^{x} & =-\frac{1}{\alpha+1}\left[\lambda e^{\sigma t-x}(a \sin y+b \cos y)\right. \\
& \left.\left((\alpha u+\beta)^{1 / \alpha}\left(\alpha u+(\alpha+1) u_{x}+\beta\right)-\beta^{1 / \alpha+1}\right)\right] \\
T^{y} & =\frac{\lambda e^{\sigma t-x}}{\alpha+1}\left[(\alpha u+\beta)^{1 / \alpha}(\alpha u+\beta)(a \cos y-b \sin y)\right. \\
& \left.-(\alpha+1) u_{y}(a \sin y+b \cos y)\right]+\frac{\lambda e^{\sigma t-x} \beta^{1 / \alpha+1}}{\alpha+1}(b \\
& \cdot \sin y-a \cos y), \\
T^{t} & =u_{t} e^{\sigma t-x}(a \sin y+b \cos y) .
\end{aligned}
$$

Notice that the last case admits the additional multipliers $e^{\sigma t} Q_{A, B}^{x}$ and $e^{\sigma t} Q_{A, B}^{y}$.

Case 7. This case admits six multipliers given by

$$
\begin{aligned}
& Q_{1}=e^{-x}(a \sin y+b \cos y), \\
& Q_{2}=e^{x}(a \sin y+b \cos y), \\
& Q_{3}=e^{\sigma t-x}(a \sin y+b \cos y), \\
& Q_{4}=e^{\sigma t+x}(a \sin y+b \cos y), \\
& Q_{5}=e^{\sigma t} Q_{A, B}^{x}, \\
& Q_{6}=e^{\sigma t} Q_{A, B}^{y} .
\end{aligned}
$$

\section{Conclusion}

In this paper, we carried out a symmetry classification of the $(2+1)$-dimensional nonlinear damped wave equation. It has been shown that the minimal subalgebra admitted by the equation is four-dimensional. In some interesting cases, the subalgebra can be extended by one to three extra symmetries. Reductions of the studied equation have also been performed using two-dimensional subalgebras where some exact solutions have been obtained. Furthermore, the multipliers have been provided for most of the cases discussed in the paper while conservation laws have been constructed for some cases of interest.

The study presented in this paper paves the way for further investigation regarding nonlinear damped wave equations arising in mathematical physics or other scientific fields. In particular the $(3+1)$ nonlinear damped wave equation could also be considered.

\section{Competing Interests}

The authors declare that there is no conflict of interests regarding the publication of this paper.

\section{References}

[1] R. G. Dean and R. A. Dalrymple, Water Wave Mechanics for Engineers \& Scientists, vol. 12 of Advanced Series on Ocean Engineering, World Scientific, 2007.

[2] C. Misner, K. Thorne, and J. Wheeler, Gravitatoin, W H Freeman, 1973.

[3] G. W. Griffiths and W. E. Schiesser, "Linear and nonlinear waves," Scholarpedia, vol. 4, no. 7, p. 4308, 2009.

[4] W. F. Bynam, E. J. Browne, and R. Porter, Dictionary of the History of Science, Princton University Press, 1984.

[5] L. Eyges, The Classical Electromagnetic Field, Dover Publications, New York, NY, USA, 1972.

[6] F. Cajori, A History of Mathematics, MacMillan, New York, NY, USA, 1961.

[7] S. J. Farlow, Partial Differential Equations for Scientists and Engineers, chapter 17, Dover, New York, NY, USA, 1993.

[8] M. J. Ablowitz, D. J. Kaup, A. C. Newell, and H. Segur, "Method for solving the sine-Gordon equation," Physical Review Letters, vol. 30, pp. 1262-1264, 1973.

[9] R. Abraham and J. E. Marsden, Foundations of Mechanics, Benjamin/Cummings, 1978.

[10] B. Fornberg and G. B. Whitham, "A numerical and theoretical study of certain nonlinear wave phenomena," Philosophical Transactions of the Royal Society of A-Mathematical, Physical Sciences and Engineering Sciences, vol. 289, no. 1361, pp. 373404, 1978.

[11] S. B. Wineberg, B. Stephen, J. F. McGrath et al., "Implicit spectral methods for wave propagation problems," Journal of Computational Physics, vol. 97, no. 2, pp. 311-336, 1991.

[12] M. Toda, Nonlinear Waves and Solitons, Kluwer Academic Publisher, 1989.

[13] H. Kumar and F. Chand, "1-Soliton solutions of complex modified KdV equation with time-dependent coefficients," Indian Journal of Physics, vol. 87, no. 9, pp. 909-912, 2013. 
[14] J. L. Yin, Q. Q. Xing, and L. X. Tian, "Melnikov analysis and chaos control of nonlinear dispersive $\mathrm{KdV}$ equation under external periodic perturbation," Indian Journal of Physics, vol. 89, no. 3, pp. 273-279, 2015.

[15] V. K. Sharma, "Chirped soliton-like solutions of generalized nonlinear Schrödinger equation for pulse propagation in negative index material embedded into a Kerr medium," Indian Journal of Physics, vol. 90, no. 11, pp. 1271-1276, 2016.

[16] M. Jradeh, "On the damped wave equation," in Proceedings of the 12th International Conference on Hyperbolic Problems, College Park, Md, USA, June 2008.

[17] B. Vick and R. L. West, "Analysis of damped waves using the boundary element method," Wet Transaction Model Simulation, vol. 15, pp. 265-278, 1997.

[18] J. A. Stratton, Electromagnetic Theory, McGraw Hill, New York, NY, USA, 1941.

[19] V. Georgiev and G. Todorova, "Existence of a solution of the wave equation with nonlinear damping and source terms," Journal of Differential Equations, vol. 109, no. 2, pp. 295-308, 1994.

[20] M. A. Rincon and M. I. Copetti, "Numerical analysis for a locally damped wave equation," Journal of Applied Analysis and Computation, vol. 3, no. 2, pp. 169-182, 2013.

[21] M. L. Gandarias, M. Torrisi, and A. Valenti, "Symmetry classification and optimal systems of a non-linear wave equation," International Journal of Non-Linear Mechanics, vol. 39, no. 3, pp. 389-398, 2004.

[22] N. H. Ibragimov, CRC Handbook of Lie Group Analysis of Differential Equations, Volume 1, Symmetries, Exact Solutions and Conservation Laws, CRC Press, Boca Raton, Fla, USA, 1994.

[23] N. H. Ibragimov, CRC Handbook of Lie Group Analysis of Differential Equations, vol. 3 of New Trends in Theoretical Developments and Computational Methods, CRC Press, Boca Raton, Fla, USA, 1995.

[24] A. H. Kara, A. H. Bokhari, and F. D. Zaman, "On the exact solutions of the nonlinear wave and $\phi 4$-model equations," Journal of Nonlinear Mathematical Physics, vol. 15, supplement 1, pp. 105-111, 2008.

[25] E. Noether, "Invariante Variationsprobleme," Nachr. Konig. Gesell. Wisen. Gottingen, Math. Phys. Kl, vol. 2, pp. 235-257, 1918.

[26] E. Noether, "Nachrichten von der Gesellschaft der Wissenschaften zu Göttingen," Mathematisch-Physikalische Klasse, vol. 1918, no. 2, pp. 235-257, 1918, English translation in Transport Theory and Statistical Physics, vol. 1, no. 3, p. 186, 1971.

[27] S. C. Anco and G. Bluman, "Direct construction of conservation laws from field equations," Physical Review Letters, vol. 78, no. 15, pp. 2869-2873, 1997.

[28] S. Anco and G. W. Bluman, "Direct construction method for conservation laws of partial differential equations Part II: general treatment," European Journal of Applied Mathematics, vol. 13, no. 5, pp. 567-585, 2002.

[29] N. Euler and W. H. Steeb, Continuous Symmetries, Lie Algebras and Differential Equations, Bibliographisches Institut, Mannheim, Germany, 1992. 


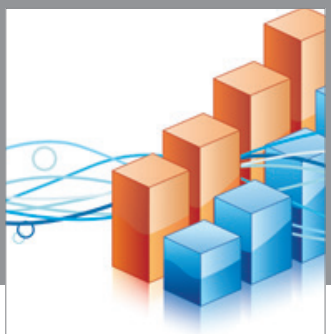

Advances in

Operations Research

vatem alat4

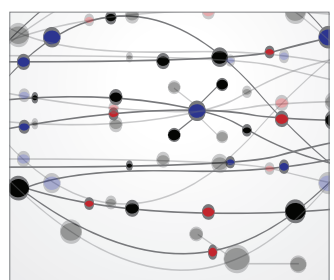

\section{The Scientific} World Journal
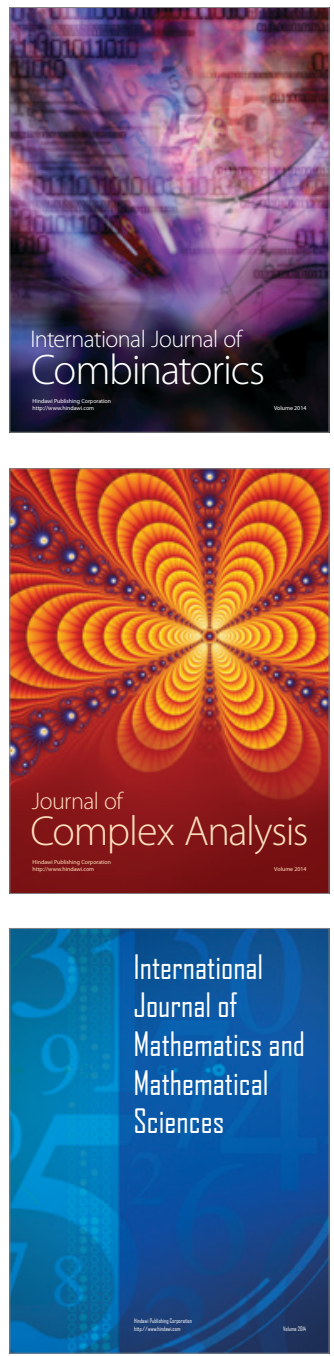
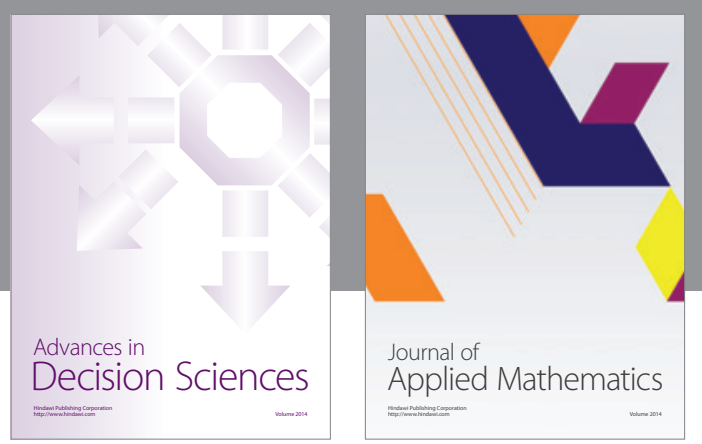

Algebra

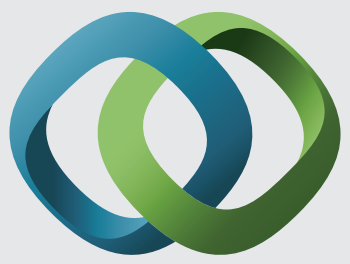

\section{Hindawi}

Submit your manuscripts at

https://www.hindawi.com
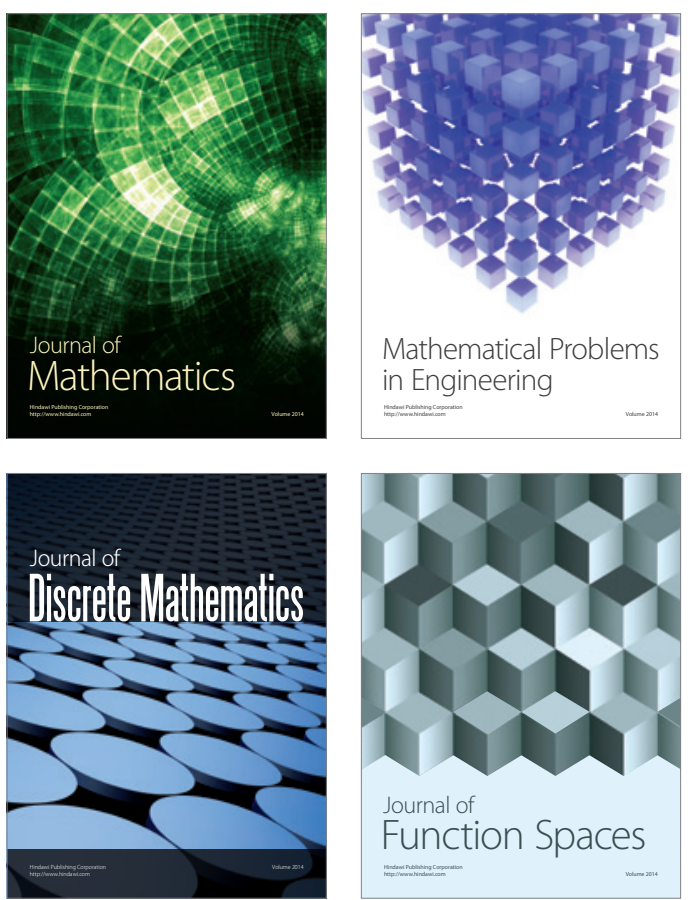

Mathematical Problems in Engineering
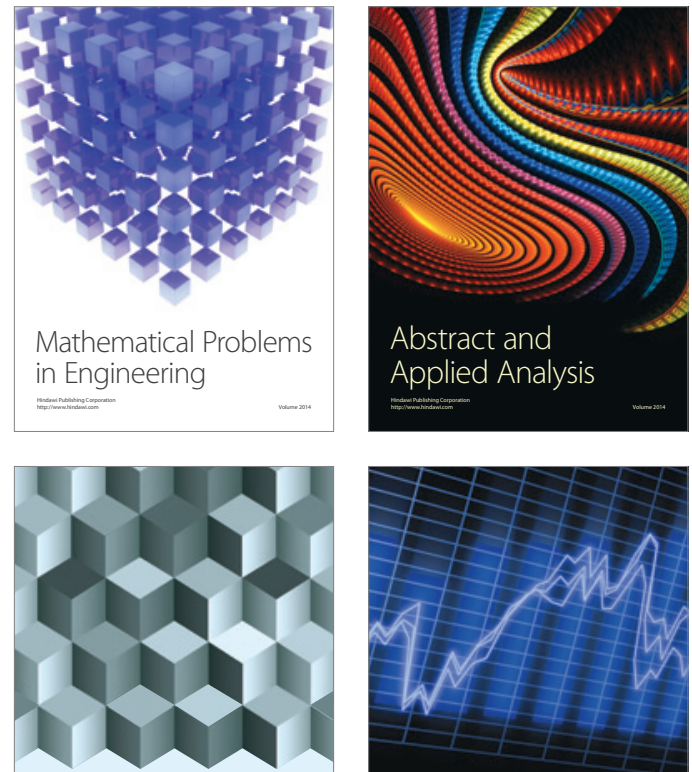

Journal of

Function Spaces

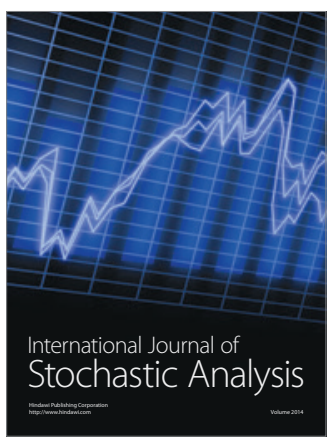

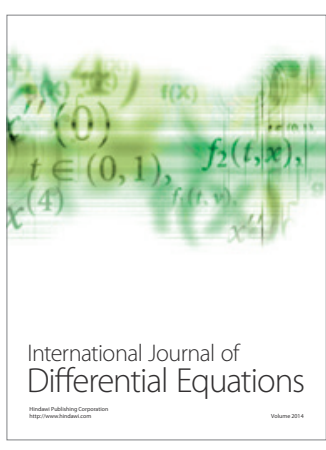
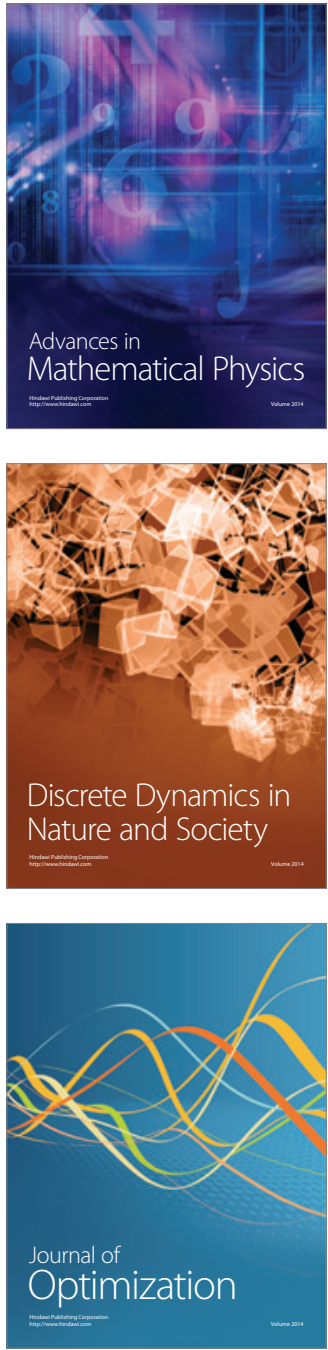